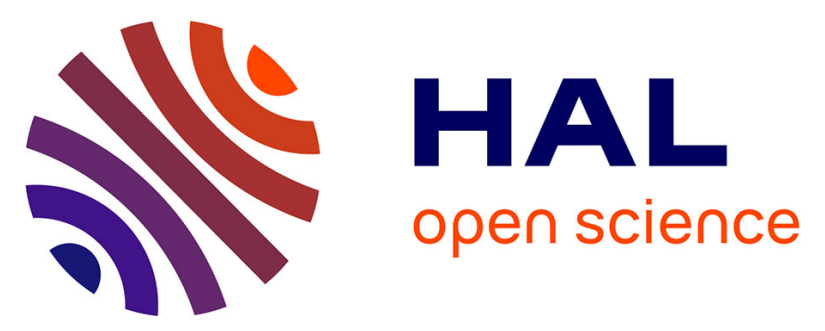

\title{
A multiscale method for transient dynamic analysis of assemblies with friction
}

David Odièvre, Pierre-Alain Boucard, Fabrice Gatuingt

\section{To cite this version:}

David Odièvre, Pierre-Alain Boucard, Fabrice Gatuingt. A multiscale method for transient dynamic analysis of assemblies with friction. 6th International Conference on Engineering Computational Technology (ECT 2008), Sep 2008, Athènes, Greece. pp.40, 10.4203/ccp.89.40 . hal-01625158

\section{HAL Id: hal-01625158 \\ https://hal.science/hal-01625158}

Submitted on 27 Oct 2017

HAL is a multi-disciplinary open access archive for the deposit and dissemination of scientific research documents, whether they are published or not. The documents may come from teaching and research institutions in France or abroad, or from public or private research centers.
L'archive ouverte pluridisciplinaire HAL, est destinée au dépôt et à la diffusion de documents scientifiques de niveau recherche, publiés ou non, émanant des établissements d'enseignement et de recherche français ou étrangers, des laboratoires publics ou privés. 


\title{
A multiscale method for transient dynamic analysis of assemblies with friction \\ D. Odièvre ${ }^{1}$, P.-A. Boucard ${ }^{1}$ and F. Gatuingt ${ }^{1}$
}

\author{
${ }^{1}$ LMT-Cachan (ENS Cachan/CNRS/Universit Paris 6/PRES UniverSud Paris) \\ 61 av. du President Wilson,F-94230 Cachan, France
}

\begin{abstract}
The aim of the present work is to develop an efficient strategy for the parametric analysis of dynamic problems with multiple contacts. The approach is based on the multiscale LATIN method with domain decomposition. We propose to take advantage of the capability of the multiscale LATIN method to reuse the solution of a given problem in order to solve similar problems. This strategy has already been applied successfully to a variety of static problems; here, it is extended to dynamics.
\end{abstract}

Keywords: multiscale computational method, transient dynamic, parametric study, domain decomposition, contact, friction.

\section{Introduction}

Modeling and simulation have an important role in engineering and design departments and raise multiple problems, particularly in dynamics in the case of large assemblies with connections. These connections play a major role in the dimensioning process because they are subject to highly nonlinear local phenomena (contact and friction) which are even more important in fast transient dynamic problems and require very fine meshes in order to be represented correctly [1]. Therefore, the choice of an appropriate and efficient computational method is of vital importance. Among the methods usually used to deal with such problems in dynamics, one can quote the two-level FETI method (often qualified dual Schur method) [2].

The aim of this work is to develop an efficient strategy for the parametric analysis of dynamic problems with multiple contacts. The applications concern elastic structural assemblies in dynamics with local nonlinearities, such as unilateral contact with friction. Our approach is based on a decomposition of an assembly into substructures and interfaces. Within each substructure, the problem is solved using the finite element method and an iterative scheme based on the multiscale LArge Time INcrement (LATIN) method [3]. The objective is to calculate a large number of design configurations [5], each of which corresponds to a set of values of all the variable parameters 
(friction coefficients, prestresses) introduced into the mechanical analysis. Here, using the capabilities of the multiscale LATIN method, instead of carrying out a full analysis for each design configuration, we propose to reuse the solution of a particular problem with one set of parameters in order to solve similar problems with other sets of parameters) [6]. The multiscale LATIN method is a mixed method which deals with both velocities and forces at the interfaces simultaneously and solves a homogenized macroscopic problem in order to accelerate the convergence of the numerical scheme.

First, we introduce the multiscale LATIN strategy for the dynamic case, focusing particularly on the construction of the "macroscopic" problem in space, which has a less conventional meaning in this case than in statics. Then, we address the details of the specific treatment of the interfaces which ensures continuity of loads and velocities. Finally, we illustrate the efficiency of the method through a parametric study of $3 \mathrm{D}$ examples.

\section{The multiscale LATIN method}

This multiscale domain decomposition method is based on three ingredients: spatial decomposition of the domain, separation of the scales and a resolution algorithm. The main features of these three ingredients are developed below. The details of the method itself can be found in [3].

\subsection{Decomposition into substructures and interfaces}

An assembly is a set of substructures which communicate with one another through interfaces, see Figure 1(a). Each interface represents a connection. The substructures and interfaces have their own variables and equations (admissibility, equilibrium and behavior). Two connected substructures are denoted $\Omega_{E}$ and $\Omega_{E^{\prime}}$ and the associated interface is designated by $\Gamma_{E E^{\prime}}$.

Each interface is a mechanical entity with its own variables and its specific behavior, which depends on the type of connection. Many different types of connections, e.g. frictional contact, can be modeled with this approach. The interface variables consist of two force fields $F_{E}, F_{E^{\prime}}$ and two dual velocity fields $W_{E}, W_{E^{\prime}}$, see Figure 1(b). By convention, $F_{E}$ and $F_{E^{\prime}}$ represent the action of the interface on the substructures, and $W_{E}$ and $W_{E^{\prime}}$ are the velocities of the substructures viewed from the interface. Thus, the interface concept can be easily extended to the boundary, where the displacements or the velocities or the forces are prescribed. 
Figure 1: Decomposition of the reference problem into substructures and interfaces

\subsection{Multiscale extension}

In order to ensure the theoretical scalability of the method, our approach introduces a spatial description of the unknowns on two scales, called the macroscale and the microscale. In this multiscale strategy, the interfaces play a major role of scale separation: the definitions of the microscopic and macroscopic fields are related to the interface quantities of the substructured problem and are expressed prior to any discretization.

Let us consider an interface $\Gamma_{E E^{\prime}}$ whose unknowns $\left(W_{E}, F_{E}\right)$ are divided into

$$
W_{E}=W_{E}^{m}+W_{E}^{M} \text { and } F_{E}=F_{E}^{m}+F_{E}^{M}
$$

, where $W^{M}$ and $W^{m}$ denote respectively the macro parts and the micro complements of the velocity field. The separation of the two scales is obtained by means of the projection operator $\Pi_{\Gamma_{E E^{\prime}}}$, defined for each interface. Over $\Gamma_{E E^{\prime}}$, we write $W^{M}$ and $F^{M}$ in the form $X^{M}=\sum\left(X, e_{i}^{M}\right) e_{i}^{M}=\Pi_{\Gamma_{E E^{\prime}}} X$. The choice of the macroscopic projector influences the efficiency of the algorithm. The selection of the optimum projector was studied in [4]. The basis functions $\left\{e_{i}^{M}\right\}$ for a 2D problem are represented in Figure 2. The macroscopic kinematics which results from this choice consists of two translations, one rotation and one strain.

Figure 2: The affine basis functions $\left\{e_{i}^{M}\right\}$ of an interface $\Gamma_{E E^{\prime}}$ 


\subsection{The substructured problem}

The problem within a substructure: The displacement field at any point $M$ of $\Omega_{E}$ and at any time $t$ of $[0, T]$ is $u_{E}(M, t)$, and the associated space is $\mathcal{U}^{[0, T]} . \varepsilon_{E}$ is the strain field and the current state of the structure is characterized by the stress field $\sigma_{E}$, whose associated space is $\mathcal{S}^{[0, T]}$. The mechanical problem to be solved within each substructure $\Omega_{E}$ is:

Find the evolutions of the displacement field $u_{E}(M, t)$ and stress field $\sigma_{E}(M, t)$ such that:

- Kinematic admissibility: $\forall t \in[O, T], u_{E} \in \mathcal{U}^{[0, T]}$

- Initial condition: $\forall M \in \Omega_{E}$

$$
u_{E}(t=0)=U_{E}^{0} \quad \frac{d u_{E}}{d t}(t=0)=V_{E}^{0}
$$

- Boundary condition: $\forall t \in[O, T], \forall M \in \Gamma_{E E^{\prime}}$

$$
\left.\frac{d u_{E}}{d t}\right|_{\Gamma_{E E^{\prime}}}=W_{E} \quad u_{\left.E\right|_{\partial \Omega_{1}}}=U_{d} \quad F_{\left.E\right|_{\partial \Omega_{2}}}=F_{d}
$$

- Equilibrium: $\forall t \in[0, T], \forall \dot{u}^{*} \in U_{0}^{[0, T]}, \sigma_{E} \in \mathcal{S}^{[0, T]}$

$$
\int_{\Omega_{E}}\left(\rho \frac{d^{2} u_{E}}{d t^{2}}+f_{d}\right) \dot{u}^{*} d \Omega+\int_{\Omega_{E}} \operatorname{Tr}\left(\sigma_{E} \varepsilon\left(\dot{u}^{*}\right)\right) d \Omega=\sum_{E^{\prime}} \int_{\Gamma_{E E^{\prime}}} F_{E E^{\prime}} \dot{u}^{*} d \Gamma
$$

- Elastic behavior: $\forall t \in[0, T], \forall M \in \Omega_{E}$

$$
\sigma_{E}=\mathbf{K}_{E} \varepsilon\left(u_{E}\right)
$$

where $\mathbf{K}_{E}$ is the Hooke's operator.

The problem at the interfaces: The mechanical problem to be solved at each interface $\Gamma_{E E^{\prime}}$ is:

Find the evolutions of the force fields $F_{E}(M, t), F_{E^{\prime}}(M, t)$ and velocity fields $W_{E}(M, t)$, $W_{E^{\prime}}(M, t)$ such that:

- Behavior: $\forall t \in[O, T], \forall M \in \Gamma_{E E^{\prime}}$

$$
\left(F_{E}, F_{E^{\prime}}\right)=A_{\Gamma_{E E^{\prime}}}\left(W_{E}, W_{E^{\prime}}\right)
$$

where the behavior is expressed as an evolution law $A_{\Gamma_{E E^{\prime}}}$. This law can be nonlinear, e.g. for frictional contact. 


\subsection{Resolution strategy: the LATIN method}

The LATIN (LArge Time INcrement) method [7] is a general, mechanics-based computational strategy for the resolution of time-dependent nonlinear problems which operates over the entire time-space domain. It has been applied successfully to a variety of problems $[10,9,8,4]$.

In our particular case of linear elastic substructures, the solution $u_{E}(M, t), \sigma_{E}(M, t)$ can be calculated from the boundary values $W_{E}(M, t), F_{E}(M, t)$. Thus, a solution $s$ is represented only by the force and velocity fields on both sides of an interface. The solution of Problem $s_{\text {ref }}$ is expressed as a set of time-dependent fields within each substructure and at the corresponding interfaces:

$$
s_{\text {ref }}=\sum_{E} s_{E} \quad s_{E}=\left\{F_{E}(M, t), W_{E}(M, t)\right\}
$$

\section{- Separation of the difficulties:}

The LATIN approach is based on the idea of dealing with each difficulty separately in order not to have to solve a global problem and a nonlinear problem at the same time. The equations are divided into global linear equations and local nonlinear equations, so that $s_{r e f}=A_{d} \cap \Gamma$ is the intersection of two subspaces:

- $A_{d}$, the space of the solutions of the linear equations associated with the substructures $\Omega_{E}$ : kinematic admissibility, equilibrium, elastic behavior and admissibility of macroquantities;

- $\Gamma$, the space of the solutions of the local equations related to the interfaces $\Gamma_{E E^{\prime}}$ and expressing their behavior.

\section{A two-step iterative strategy:}

The LATIN method consists in seeking fields of $\Gamma$ and $A_{d}$ alternatively in two search directions $E^{+}$and $E^{-}$, as shown in Figure 3. Each iteration involves two stages, called the local stage and the linear stage:

Local stage: given $s_{n} \in A_{d}$, find $\widehat{s}$ such that:

$$
\begin{array}{cccc}
\widehat{s}_{n+1 / 2} & \in & \Gamma & \text { (interfaces) } \\
\widehat{s}_{n+1 / 2}-s_{n} & \in & E^{+} & \text {(search directions) }
\end{array}
$$

Linear stage: given $\widehat{s} \in \Gamma$, find $s_{n+1}$ such that:

$$
\begin{array}{cccc}
s_{n+1} & \in & A_{d} & \text { (substructures) } \\
s_{n+1}-\widehat{s}_{n+1 / 2} & \in & E^{-} & \text {(search directions) }
\end{array}
$$

In our particular case of linear elastic substructures, the search directions are defined as follows:

$$
\begin{array}{ccc}
\widehat{s}_{n+1 / 2}-s_{n} \in E^{+} & \Longleftrightarrow \widehat{F}_{E}-F_{E}=\mathbf{k}_{0}\left(\widehat{W}_{E}-W_{E}\right) \\
s_{n+1}-\widehat{s}_{n+1 / 2} \in E^{-} & \Longleftrightarrow F_{E}-\widehat{F}_{E}=-\mathbf{k}_{0}\left(W_{E}-\widehat{W}_{E}\right)
\end{array}
$$


Figure 3: An iteration of the LATIN method

where $\mathbf{k}_{0}$ is a scalar parameter of the method. As long as $\mathbf{k}_{0}$ is positive, the solution of the problem does not depend on the value of this parameter, which affects only the convergence rate of the algorithm. In the dynamic cases which we are studying here, the optimum value of $\mathbf{k}_{0}$ for a $1 \mathrm{D}$ problem is given by [10]:

$$
\mathbf{k}_{0}=\sqrt{\rho E}
$$

where $E$ is the Young's modulus and $\rho$ the density. $\mathbf{k}_{0}$ can be interpreted as a local impedance of the material.

An error indicator $\eta$ is used to control the convergence of the algorithm. This indicator is a measure of the distance between the two solutions $s_{n+1}$ and $\widehat{s}_{n+1 / 2}$ :

$$
\eta=\frac{\sum_{E}\left\|s_{n+1}-\widehat{s}_{n+1 / 2}\right\|^{2}}{\sum_{E}\left\|s_{n+1}\right\|^{2}+\sum_{E}\left\|\widehat{s}_{n+1 / 2}\right\|^{2}}
$$

where: $\left\|s_{n+1}\right\|_{E}^{2}=\int_{0}^{T} \int_{\partial \Omega_{E}} F_{E}^{T} \mathbf{k}_{0}^{-1} F_{E}+W_{E} \mathbf{k}_{0} W_{E} d S d t$

\subsection{The local stage: $\widehat{s}_{n+1 / 2}$}

This stage consists in building $\widehat{s}_{n+1 / 2} \in \Gamma$ knowing $s_{n} \in A d$. Then, $\left(\widehat{s}_{n+1 / 2}-s_{n}\right)$ must follow the search direction $E^{+}$defined as follows:

$$
\left(\widehat{F}_{E}-F_{E}\right)-\mathbf{k}_{0}\left(\widehat{W}_{E}-W_{E}\right)=0
$$

Let us consider the case of a perfect interface $\Gamma_{E^{\prime} E}$. The unknowns are $\left(\widehat{W}_{E}, \widehat{W}_{E^{\prime}}, \widehat{F}_{E}, \widehat{F}_{E^{\prime}}\right)$ and must verify the behavior equation:

$$
\widehat{F}_{E}+\widehat{F}_{E^{\prime}}=0 \quad \widehat{W}_{E}=\widehat{W}_{E^{\prime}}
$$

The solution of Equations 9 and 10 is:

$$
\begin{gathered}
\widehat{W}_{E}=\underline{W}_{E^{\prime}}=\frac{1}{2}\left(\underline{W}_{E}+\underline{W}_{E^{\prime}}\right)-\frac{1}{2 \mathbf{k}_{0}}\left(\underline{F}_{E}+\underline{F}_{E^{\prime}}\right) \\
\underline{\widehat{F}}_{E}=-\underline{\widehat{F}}_{E^{\prime}}=\frac{1}{2}\left(\underline{F}_{E}-\underline{F}_{E^{\prime}}\right)-\frac{\mathbf{k}_{0}}{2}\left(\underline{W}_{E}-\underline{W}_{E^{\prime}}\right)
\end{gathered}
$$


The local stage consists in solving local problems at this interface. The case of more complex interfaces (contact, friction,...) was developed in [4].

\subsection{The linear stage: $s_{n+1}$}

This stage consists in building $s_{n+1} \in A d$ knowing $\widehat{s}_{n+1 / 2} \in \Gamma$ :

- Macro admissibility: in order to ensure the admissibility conditions of the macro variables, we introduce Lagrange multipliers $\widetilde{W}_{E}^{M}$ at the interfaces.

- Search direction: the unknowns $\left(W_{E}, F_{E}\right)$ must follow the search direction. Equation 7 must be modified by introducing the Lagrange multipliers defined at the interfaces. The new search direction $E^{-}$is defined as follows:

$$
\left(F_{E}-\widehat{F}_{E}\right)+\mathbf{k}_{0}\left(W_{E}-\widehat{W}_{E}-\widetilde{W}_{E}^{M}\right)=0
$$

- Equations associated with the substructures: the unknowns $\left(W_{E}, F_{E}\right)$ must verify the dynamic equilibrium (3) and elastic behavior (4) equations.

These equations lead to the resolution of an independent problem, called the "micro" problem, in each substructure:

Find $u_{E}(M, t), \forall t \in[0, T], \forall \dot{u}^{*} \in U_{0}^{[0, T]}, \sigma_{E} \in \mathcal{S}^{[0, T]}$

$$
\begin{array}{r}
\int_{\Omega_{E}}\left(\rho \frac{d^{2} u_{E}}{d t^{2}}+f_{d}\right) \dot{u}^{*} d \Omega+\int_{\partial \Omega_{E}} \mathbf{k}_{0} \frac{d u_{E}}{d t} \dot{u}^{*} d S+\int_{\Omega_{E}} \mathbf{K}_{E} \varepsilon\left(u_{E}\right) \varepsilon\left(\dot{u}^{*}\right) d \Omega= \\
\sum_{E^{\prime}} \int_{\Gamma_{E E^{\prime}}}\left(\widehat{F}_{E}+\mathbf{k}_{0} \widehat{W}_{E}+\mathbf{k}_{0} \widetilde{W}_{E}^{M}\right) \dot{u}^{*} d \Gamma
\end{array}
$$

- Discretization: In each substructure, using a classical finite element discretization $u_{E}(M)=\{N\}^{T}\{U\}$ and $\varepsilon_{E}(M)=[B]\{U\}$, Equation 14 leads to the resolution of an evolution problem: Find $U(M, t), \forall t \in[O, T]$ such that:

$$
\left[M_{E}\right] \ddot{U}(t)+\left[c_{E}\right] \dot{U}(t)+\left[K_{E}\right] U(t)=\widehat{F}+\mathbf{k}_{0}\left(\widehat{W}+\widetilde{W}^{M}\right)
$$

where $\left[M_{E}\right]$ and $\left[K_{E}\right]$ are the classical finite element mass and stiffness matrices. Matrix $\left[c_{E}\right]$ is less classical and due only to the LATIN method. These matrces are defined by:

$$
\begin{aligned}
M_{E} & =\int_{\Omega_{E}} \rho\{N\}^{T}\{N\} d \Omega \\
c_{E} & =\int_{\partial \Omega_{E}} \mathbf{k}_{0}\{N\}^{T}\{N\} d S \\
K_{E} & =\int_{\Omega_{E}}[B] \mathbf{K}_{E}[B] d \Omega
\end{aligned}
$$

In order to solve the evolution problem (15), the finite element discretization must be associated with an explicit time integration scheme. We chose to use the classical 
central difference scheme:

$$
\begin{aligned}
\dot{U}_{t+\Delta t} & =\dot{U}_{t}+\frac{\Delta t}{2}\left(\ddot{U}_{t}+\ddot{U}_{t+\Delta t}\right) \\
U_{t+\Delta t} & =U_{t}+\Delta t \dot{U}_{t}+\frac{\Delta t^{2}}{2} \ddot{U}_{t}
\end{aligned}
$$

Then, the linear system which needs to be solved at each time step has the following form:

$$
\begin{array}{r}
\left(\frac{2}{\Delta t}\left[M_{E}\right]+\left[c_{E}\right]\right) \dot{U}_{t+\Delta t}=\widehat{F}_{t+\Delta t}+\mathbf{k}_{0}\left(\widehat{W}_{t+\Delta t}+\widetilde{W}_{t+\Delta t}^{M}\right)-\left[K_{E}\right] U_{t} \\
+\left(\frac{2}{\Delta t}\left[M_{E}\right]-\Delta t\left[K_{E}\right]\right) \dot{U}_{t}+\left(\left[M_{E}\right]-\frac{\Delta t^{2}}{2}\left[K_{E}\right]\right) \ddot{U}_{t}
\end{array}
$$

We use a lumped mass matrix $\left[M_{E}\right]$, and $\left[c_{E}\right]$ is also a diagonal matrix. System (18) cannot be solved because there are two unknowns, $\dot{U}_{t+\Delta t}$ and $\widetilde{W}_{t+\Delta t}^{M}$. Therefore, we divide Field $\dot{U}$ into two fields, $\dot{U}^{1}$ and $\dot{U}^{2}$, such that $\dot{U}=\dot{U}^{1}+\dot{U}^{2}$ and fields $\dot{U}^{1}$ and $\dot{U}^{2}$ are solutions of two microproblems:

$$
\begin{aligned}
\left(\frac{2}{\Delta t}\left[M_{E}\right]+\left[c_{E}\right]\right) \dot{U}_{t+\Delta t}^{1} & =\widehat{F}_{t+\Delta t}+\mathbf{k}_{0} \widehat{W}_{t+\Delta t}+f\left(\ddot{U}_{t}, \dot{U}_{t}, U_{t}\right) \\
\left(\frac{2}{\Delta t}\left[M_{E}\right]+\left[c_{E}\right]\right) \dot{U}_{t+\Delta t}^{2} & =\mathbf{k}_{0} \widetilde{W}_{t+\Delta t}^{M}
\end{aligned}
$$

Equation 19 can be easily solved after a local stage. Equation 20, however, cannot be solved without the knowledge of $\widetilde{W}^{M}$. This linear system (20) can be easily inverted because of the very small number of degrees of freedom of $\widetilde{W}^{M}$ (four DOFs per interface for a $2 \mathrm{D}$ problem). Thus, we can write:

$$
W^{2, M}=\mathbf{L}_{E}^{-1} \widetilde{W}^{M}
$$

where $W^{2, M}=\left.\Pi_{\Gamma_{E E^{\prime}}} \dot{U}^{2}\right|_{\Gamma_{E E^{\prime}}}$

and $\mathbf{L}_{E}$ represents a homogeneous behavior operator for Substructure $\Omega_{E}$. These operators are calculated only once for all the substructures at the beginning of the algorithm. $W^{2, M}$ is the macro part of the restriction of $\dot{U}^{2}$ to the interfaces.

The macro problem: The admissibility of the macroquantities at all the interfaces and the homogeneous behavior of all the substructures (Equation 21) lead to the definition of the "macro" problem.

In order to explain the construction of the macro problem, let us consider a perfect interface. The admissibility of the macroquantities at such an interface corresponds to the continuity of the velocities and to the equilibrium of the macroscopic forces. For an interface $\Gamma_{E E^{\prime}}$, one has:

$$
W_{E}^{M}=W_{E^{\prime}}^{M} \quad \text { and } \quad F_{E}^{M}+F_{E^{\prime}}^{M}=0
$$


The decomposition of Field $\dot{U}$ into $\dot{U}^{1}+\dot{U}^{2}$ using the search direction (13) and the projector $\Pi_{\Gamma_{E E^{\prime}}}$ involves some other relations for $W^{1, M}$ and $W^{2, M}$ (the macro parts of the restrictions of fields $\dot{U}^{1}$ and $\dot{U}^{2}$ to the interfaces):

$$
\begin{array}{r}
W^{M}=W^{1, M}+W^{2, M} \\
F^{1, M}-\widehat{F}^{M}+\mathbf{k}_{0}\left(W^{1, M}-\widehat{W}^{M}\right)=0 \\
F^{2, M}+\mathbf{k}_{0}\left(W^{2, M}-\widetilde{W}^{M}\right)=0
\end{array}
$$

With such admissibility conditions, we need to introduce two Lagrange multipliers for each interface, as shown in Figure 4.

Figure 4: Lagrange multipliers $\widetilde{W}_{E}^{M}$ and $\widetilde{W}_{E^{\prime}}^{M}$ for a perfect interface

The contribution of the perfect interface $\Gamma_{E E^{\prime}}$ to the macro problem (Equation 24) is expressed through Equations 21, 22 and 23. The quantity $\widetilde{W}_{E}^{M}$ is a vector which contains all the Lagrange multipliers of Substructure $\Omega_{E}$.

$$
\left[\begin{array}{cc}
\mathbf{L}_{E} & -\mathbf{L}_{E^{\prime}} \\
\mathbf{k}_{0}\left(1-\mathbf{L}_{E}\right) & \mathbf{k}_{0}\left(1-\mathbf{L}_{E^{\prime}}\right)
\end{array}\right]\left[\begin{array}{c}
\widetilde{W}_{E}^{M} \\
\widetilde{\widetilde{W}}_{E^{\prime}}^{M}
\end{array}\right]=\left[\begin{array}{c}
-W_{E}^{1, M}+W_{E^{\prime}}^{1, M} \\
-F_{E}^{1, M}-F_{E^{\prime}}^{1, M}
\end{array}\right]
$$

This problem couples all the macro variables of the entire structure and enables us to define the Lagrange multiplier $\widetilde{W}_{E}^{M}$ for all the substructures. $W_{E}^{1, M}$ is the macro part of the solution of the first microproblem (Equation 19); $F_{E}^{1, M}$ is calculated using the search direction (Equation 23).

\subsection{The algorithm}

The LATIN method consists in processing linear and local stages alternatively. Algorithm 1 shows the key steps of an iteration of the multiscale method. 


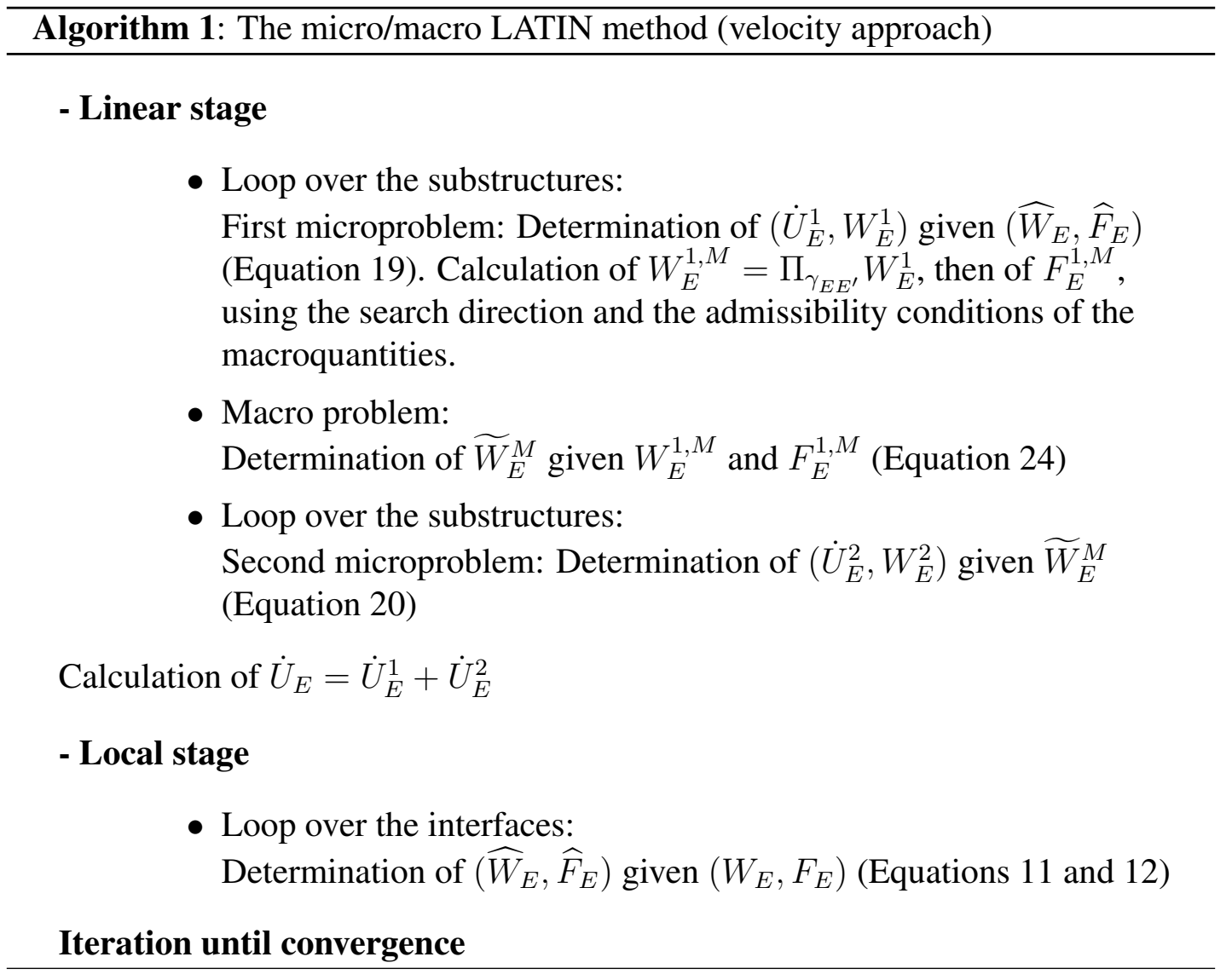

\subsection{Strategy for the parametric study}

At each iteration, the LATIN method leads to an approximate solution of the problem over the whole time interval. The idea consists in reusing this approximation to find the solution of a problem similar to that for which it was calculated in the first place. The multiple-solution method uses the fact that the LATIN algorithm can be initialized with any solution (usually an elastic solution) provided that it verifies the admissibility conditions. Therefore, the key to our technique consists in initializing the process associated with the similar problem (the "perturbed" structure) using the results of the calculation carried out previously on the "initial" structure. Thus, a first approximation of the solution of the perturbed problem (with strong mechanical content) becomes immediately available. This idea has already been applied successfully to a variety of static and quasi-static problems [6]. In this work, we use it in the context of the multiscale LATIN method in dynamics.

In our particular case of contact between elastic structures, the interfaces play a vital role: they enable one to initiate the calculation of the perturbed structures without having to save all the data of the substructures first, and to search for the solution of the perturbed problem using an initial solution adapted to the target problem. In the 
best-case scenario, only a few iterations are necessary and the solution is obtained inexpensively. If the solutions of the "initial" and "perturbed" problems are close enough, the latter can still be derived at significantly lower cost than by using a full calculation. For a simple parametric study, the parameters change only between iterations. Thus, each calculation is initialized by the solution of the previous one. The algorithm of the full parametric study is summarized in Algorithm 2.

Algorithm 2: Parametric study with the LATIN Method

Initialization

- Loop over the substructures:

Construction and factorization of $\left[M_{E}\right],\left[c_{E}\right],\left[K_{E}\right]$.

- Loop over the interfaces:

$\widehat{W}_{E}=\widehat{W}_{E^{\prime}}=W_{E}=W_{E^{\prime}}=0$

$\widehat{F}_{E}=\widehat{F}_{E^{\prime}}=F_{E}=F_{E^{\prime}}=0$

Definition of the limits of the parameter sets

Loop $k=1,2, \ldots$ number of parameter sets

- Restoration of the quantities at the interfaces

- Iteration until convergence:

Global stage

Local stage

Convergence test

- Save the interface solution for the $k^{\text {th }}$ parameter set

\section{Examples: 3D academic example}

In order to test the method previously developed, let us consider a 3D case. This example concerns the propagation of a compression wave in a bar composed of two parallelepipeds (Figure 5). Each parallelepiped can be decomposed into several substructures. The interface between the two parallelepipeds can be perfect or can involve frictional contact. The bar is $1 \mathrm{~m}$-long and $0.25 \mathrm{~m}$-wide. Its Young's modulus is 200 $\mathrm{GPa}$, the mass density $7800 \mathrm{~kg} / \mathrm{m}^{3}$, and Poisson's ratio 0.3 . The loading consists of a prescribed velocity going from 0 initially to a maximum value of $1 \mathrm{~m} / \mathrm{s}$ over a period of $60 \mu \mathrm{s}$. 
Figure 5: Numerical example

\subsection{Comparison between the single-scale and multiscale methods}

The purpose of the test was to evaluate the efficiency of the multiscale method. In order to do that, we assumed a perfect interface between the two parallelepipeds and decomposed each parallelepiped into 2 substructures. Figure 6 shows the mesh used for the test, which contains about 5,600 DOFs. We used 79 time step of $5 \mu$ s each for a total duration of $395 \mu \mathrm{s}$.

Figure 6: The mesh used for the bar

Figure 7 shows the velocity field along the $(O, x)$ axis (defined in Figure 5) as a function of time. Using the single-scale method (Figures 7(a) and 7(b)), iterations are necessary for the wave due to the applied velocity to propagate through the structure.

(a) Single-scale method for Iteration 5 (b) Single-scale method for Iteration 10 (c) Multiscale method for Iteration 1

Figure 7: Velocity field $\mathrm{V}$ along the $(O, x)$ axis $(\mathrm{m} / \mathrm{s})$ vs. time $(\mu \mathrm{s})$ and $\mathrm{x}(\mathrm{cm})$

In the case of the multiscale method (Figure 7(c)), the first iteration of the method gives a good estimate of the solution, thanks to the macroscopic problem which pro- 
vides a representation of the macroscopic part of the solution.

The efficiency of the multiscale method is illustrated by the evolution of the error indicator during the process. Figure 8 shows the convergence rate of the single-scale and multiscale methods using 4 and 24 substructures.

Figure 8: Evolution of the error indicator during the process

These curves show that the convergence rate of the multiscale method is better than that of the single-scale method. Moreover, the convergence rate of the multiscale method does not depend on the number of substructures. The multiscale approach is scalable, whereas the convergence rate of the single-scale method is highly dependent on the number of substructures.

\subsection{Parametric study}

In order to illustrate the efficiency of the method in carrying out parametric studies, let us consider the same example, this time with frictional contact at the interface between the two parallelepipeds. In the following parametric study, the friction coefficient $f$ is assumed to be known and takes 11 different values between 0 and 0.5 in increments of 0.05 . Figure 9 shows the calculation cost for each value of $f$, which indicates that the strategy is efficient: 565 iterations were required for 11 different values of the parameter, compared to 4,345 iterations using the direct approach.

\begin{tabular}{cccccccc}
\hline $\mathrm{f}$ & 0 & 0.05 & 0.1 & 0.15 & 0.2 & 0.25 & $0.3 . .0 .5$ \\
\hline Nb. of iterations & 395 & 56 & 36 & 28 & 21 & 12 & $\approx 4$ \\
\hline Time (normalized) & 1. & 0.14 & 0.09 & 0.07 & 0.05 & 0.03 & $<0.01$ \\
\hline
\end{tabular}

Figure 9: Cost of the parametric calculations

Figure 10 shows the evolution of the error indicator during the process. Each change in $f$ increases the error indicator, but the number of iterations required decreases. 
Figure 10: Evolution of the error indicator during the parametric study

\subsection{Frictional contact with a gap}

In the previous parametric study, the parameter set consisted of only the friction coefficient $f$ between the two parallelepipeds. This example enabled us to study the influence of this coefficient on the solution. In such a study, the contact conditions must be verified strictly.

The objective of this section is to show that the multiscale LATIN method is indeed capable of carrying out such a calculation. In order to do that, let us consider the same example of two parallelepipeds with a frictional contact interface, this time with an initial $40-\mu \mathrm{m}$ gap between them. The gap between the two parallelepipeds creates a shock wave when they come in contact. We analyzed this problem with both the multiscale LATIN method and the finite element code LS-DYNA3D.

(a) Multiscale LATIN method

(b) LS-DYNA3D

Figure 11: Displacement of a point on either side of the interface as a function of time

Figure 11 shows the displacement of a point on either side of the interface with the gap as a function of time. In the result obtained with the multiscale LATIN method (Figure 11(a)), the behavior of the interface is verified: the gap is visible at the beginning of the curve; then, the two parallelepipeds come into contact. In the result obtained with LS-DYNA3D (Figure 11(b)), the behavior of the interface is not verified exactly. 
One can see that sometimes the two parallelepipeds are not in contact as they should be, and that they interpenetrate at the end of the calculation. These results show that, contrary to LS-DYNA3D, the multiscale LATIN method is suitable for studying the influence of the friction coefficient on the solution.

\section{Conclusion}

As was already proven in statics, the extension of the multiscale LATIN method to dynamics which we study in this work marks a real advantage compared to the singlescale method. The algorithm, associated with an explicit time integration scheme, is very efficient.

The strategy for parametric studies already used in statics also gives good results in dynamics. Compared to other commercial finite element tools, this solution is particularly well-adapted to the study of friction coefficients. The strategy proposed here, associated with the multiscale approach, is very efficient and can also be fully parallelized. The next step will consist in implementing this strategy within a cluster architecture and evaluating its efficiency for problems with very large numbers of degrees of freedom.

\section{References}

[1] J.O. Hallquist, G.L. Goudreau and D.J. Benson, "Sliding interfaces with contactimpact in large-scale lagrangien computations". Computer Methods in Applied Mechanics and Engineering, 51, 107-137, 1985.

[2] C. Farhat, J. Mandel, "The two-level FETI method for static and dynamic plate problems", Computer Methods in Applied Mechanics and Engineering, 555, 129-151, 1998.

[3] P. Ladevèze, O. Loiseau and D. Dureissex, "A micro-macro and parallel computational strategy for highly heterogeneous structures". Int Jal for Numerical Methods in Engineering, 52, 121-138, 2001.

[4] P. Ladevèze, A. Nouy, O. Loiseau, "A multiscale computational approach for contact problems", Computer Methods in Applied Mechanics and Engineering Sofware, 191, 4869-4891, 2002.

[5] K. S. Krishnamurthy, P. Mahajan and R. K. Mittal, "A parametric study of the impact response and damage of laminated cylindrical composite shells. Composites Science and Technology, 61, 1655-1669, 2001.

[6] P.A. Boucard and L. Champaney, "A suitable computational strategy for the parametric analysis of problems with multiple contact". Int Jal for Numerical Methods in Engineering, 57, 1259-1282, 2003.

[7] P. Ladevèze, "Nonlinear computational structural mechanics - New approaches and non-incremental methods of calculation”, Springer-Verlag, 1999. 
[8] P. Ladevèze, A. Nouy, "On a multiscale computational strategy with time and space homogenization for structural mechanics, Computer Methods in Applied Mechanics and Engineering, 192, 3061-3087, 2003.

[9] P.A. Boucard, P. Ladevèze and H. Lemoussu, "A modular approach to 3D impact computation with frictional contact”, Computer \& Structures, 78, 45-52, 2000.

[10] H. Lemoussu, P.A. Boucard, P. Ladevèze, "A 3D shock computational strategy for real assembly and shock attenuator”, Adv. Engrg. Software, 33, 517-526, 2002. 